\title{
Unfavorable Shimada Classification
}

National Cancer Institute

\section{Source}

National Cancer Institute. Unfavorable Shimada Classification. NCI Thesaurus. Code C85415.

Patients of any age with stroma-poor tumors, undifferentiated or differentiated neuroblasts, and a mitosis-karyorrhexis index of more than 200; or patients older than 18 months with stroma-poor tumors, undifferentiated neuroblasts, and a mitosiskaryorrhexis index of 100-200; or patients older than 60 months with stroma-poor tumors, differentiated neuroblasts, and a mitosis-karyorrhexis index of less than 100. 